\title{
Síndrome antisintetasa. Manejo anestésico de una entidad poco frecuente
}

\author{
Marta Errazquin-Aguirre*, Oscar González-Larrocha, Sorkunde Telletxea-Benguria y \\ Beatriz Del-Val-Villanueva \\ Servicio de Anestesiología y Reanimación, Hospital Galdakao-Usansolo, Galdakao, Vizcaya, España
}

\section{Resumen}

Objetivo: Describir tanto la técnica anestésica como las consideraciones a tener en cuenta en el manejo anestésico de un paciente con síndrome antisintetasa. Material y métodos: Se describe el método utilizado para anestesiar a un paciente con síndrome antisintetasa sometido a hemicolectomía derecha. Resultados: El empleo de una anestesia combinada junto con dosis bajas de relajantes musculares ha demostrado ser segura en una paciente con síndrome antisintetasa para cirugía abdominal. Discusión: El síndrome antisintetasa es un trastorno autoinmune incluido entre las miopatías inflamatorias idiopáticas que se caracteriza por presentar miopatía inflamatoria idiopática, afección intersticial pulmonar, manos de mecánico, artritis no erosiva y anticuerpos antisintetasa. En estos pacientes, es preferible el empleo de anestesia regional y, en caso de no ser posible, anestesia combinada. Conclusión: Los pacientes con miopatías se benefician de anestesia regional, pero en el caso de que no sea posible el empleo de esta técnica, el uso de anestesia combinada, con bajas dosis de relajantes musculares no despolarizantes, ha demostrado ser segura.

Palabras clave: Síndrome antisintetasa. Manejo anestésico. Anestesia combinada. Analgesia epidural.

\section{Antsynthetase syndrome. Anesthetic management of a rare entity}

\begin{abstract}
Objectives: To describe the anesthetic technic as the issues to take into account in the anesthetic management of a patient with antisynthetase syndrome. Materials and methods: It has been described the way to anesthetize a patient with antisynthetase syndrome undergoing right hemicolectomy. Results: The use of a combined anesthesia along with low doses of muscle relaxants have demonstrated to be safe in a patient with antisynthetase syndrome undergoing abdominal surgery. Discussion: The antisynthetase syndrome is an autoimmune disorder included in idiopathic inflammatory myopathies, and is characterized by presenting pulmonary interstitial involvement, mechanical hands, non-erosive arthritis and antisynthetase antibodies. In this patients, is preferred the use of regional anesthesia and if it is not possible, the use of combined anesthesia. Conclusion: The patients with myopathies benefit from regional anesthesia, but in case this technic wouldn't be possible, the use of combined anesthesia with low doses of non-despolarizing muscle relaxants has demonstrated to be safe.
\end{abstract}

Key words: Antisynthetase syndrome. Anesthetic management. Combined anesthesia. Epidural analgesia.

Correspondencia:

*Marta Errazquin-Aguirre

E-mail: merrazquin.1@alumni.unav.es
Fecha de recepción: 26-01-2019

Fecha de aceptación: 18-06-2019

DOI: 10.24875/RAA.19000029
Disponible en internet: 22-11-2019 Rev Argent Anestesiol. 2019;77(1):22-24 www.revistaargentinaanestesiologia.com un artículo open access bajo la licencia CC BY-NC-ND (http://creativecommons.org/licenses/by-nc-nd/4.0/). 


\section{Introducción}

El síndrome antisintetasa en una patología incluida entre las miopatías inflamatorias idiopáticas que se caracteriza por presentar anticuerpos antisintetasa en el suero. Las diferentes entidades incluidas dentro de las miopatías inflamatorias (dermatomiositis [DM], polimiositis [PM] y miositis por cuerpos de inclusión) son enfermedades autoinmunes, sistémicas, que cursan con debilidad muscular y elevación de enzimas musculares en suero ${ }^{1}$. Dentro de este grupo, caracterizamos el síndrome antisintetasa por la presencia de anticuerpos antisintetasa (el más frecuente es el anti-Jo-1), miopatía inflamatoria, manos de mecánico, enfermedad pulmonar intersticial, artritis, fiebre y fenómeno de Raynaud ${ }^{2}$.

Presentamos el caso de un paciente al que se le realizó una hemicolectomía derecha por una neoplasia de colon derecho donde se utilizó anestesia combinada con catéter epidural, siguiendo las directrices para pacientes con miopatía debido a la ausencia de bibliografía específica.

\section{Caso clínico}

Varón de 63 años, con riesgo American Society of Anesthesiologists (ASA III), fumador activo con un índice acumulado de 30 paquetes/año. Presenta criterios clínicos de bronquitis crónica simple sin afectación espirométrica ni necesidad de tratamiento, en seguimiento por el servicio de neumología. Dislipémico sin tratamiento. En el año 2010 fue diagnosticado de síndrome antisintetasa, presentando debilidad muscular proximal y pérdida de fuerza en ambas manos, con edema bilateral, sin evidencia de neumopatía intersticial asociada. Estuvo en tratamiento con corticoides durante 6 meses previos a la intervención quirúrgica, y en el momento de la cirugía estaba en situación de remisión. Sigue control por el servicio de reumatología. En 2013 se le realizó una ecocardiografía de estrés para isquemia miocárdica, que fue negativa.

El paciente acudió a quirófano en condiciones óptimas para la intervención quirúrgica. Se optó por una técnica combinada con catéter epidural torácico, con aguja Tuohy $18 \mathrm{G}$, situado en T9-T10, con una perfusión continua de levobupivacaína $0.1 \%$ más fentanilo $3 \mathrm{mcg} / \mathrm{ml}$. Se realizó intubación orotraqueal (IOT) con anestesia tópica de la vía aérea con lidocaína al $2 \%$ con una cánula de Mad-Magic, asociando anestesia general con desfluorano (0.6 MAC), remifentanilo a través de infusión controlada por objetivos $(\mathrm{TCl})$ a $3-4 \mathrm{ng} / \mathrm{ml}$ y rocuronio a dosis bajas $(0.3 \mathrm{mg} / \mathrm{kg})$, consiguiéndose a los 3 minutos unas excelentes condiciones para la IOT, con una respuesta de 1 en el tren de 4, realizándose sin incidencias. A los 35 - 40 minutos de la IOT, se objetiva una recuperación completa de la relajación muscular, a pesar de lo cual se consiguen unas condiciones óptimas quirúrgicas, con un neumoperitoneo a baja presión $(10-11 \mathrm{cmH} 20)$, y fueron necesarias únicamente pequeñas dosis de rocuronio, $5 \mathrm{mg}$, en momentos puntuales, como disección del ángulo hepático, extracción de la pieza a través de minilaparotomía, reintroducción de asas intestinales tras realizar anastomosis extracorpórea y, por último, para la realización de relaparoscopia final para el control de hemostasia, y la dosis total de rocuronio fue de $50 \mathrm{mg}$. Durante las primeras 2 horas de la intervención, la perfusión por el catéter epidural fue de $10 \mathrm{ml} / \mathrm{h}$, que se redujo a $7 \mathrm{ml} / \mathrm{h}$ hasta finalizar la intervención.

La duración total de la cirugía fue de 240 minutos, con una pérdida hemática aproximada de unos $250 \mathrm{ml}$. Se administraron $500 \mathrm{ml}$ de coloides y $500 \mathrm{ml}$ de cristaloides, y se mantuvo hemodinámicamente estable en todo momento.

Tras finalizar la cirugía, se comprobó la presencia de 3 respuestas en tren de 4, por lo que se administraron $2 \mathrm{mg} / \mathrm{kg}$ de sugammadex, y se obtuvo en 1 minuto la recuperación completa con un T4/1 mayor del $90 \%$. Se procedió a la educción anestésica y a la extubación, que resultó sin incidencias.

Una vez finalizada la intervención se trasladó al paciente a la Unidad de Reanimación, donde permaneció durante $24 \mathrm{~h}$ sin presentar complicaciones.

\section{Discusión}

Las miopatías inflamatorias idiopáticas comprenden un grupo de entidades crónicas de carácter autoinmune, como la PM, la DM y la miositis por cuerpos de inclusión. Son enfermedades autoinmunitarias sistémicas que cursan con debilidad muscular y elevación de enzimas musculares en suero ${ }^{1}$. La afectación cutánea, articular, pulmonar y de otros órganos determinan las diferentes presentaciones clínicas. La presencia de anticuerpos antinucleares es frecuente en estas miopatías inflamatorias, y se observan en un 25 - 35\% de los pacientes con PM o DM. La presencia de anticuerpos antisintetasa, como el anti-Jo-1, anti-PL-7, anti-PL-12 o anti-EJ, entre otros, es lo que define el síndrome antisintetasa, y lo más frecuente es encontrar anticuerpos anti-Jo1. En este caso, las manifestaciones clínicas son similares a las de la PM o DM, asociando, habitualmente, enfermedad pulmonar intersticial, lo que confiere un peor pronóstico ${ }^{3}$.

Los pacientes con miopatías idiopáticas asocian un mayor riesgo anestésico debido a la afectación 
muscular, cardíaca o respiratoria que puedan tener ${ }^{4}$. Po ello, debemos considerarla como una enfermedad multisistémica con repercusión sobre el acto anestésico.

Ante la ausencia de bibliografía específica sobre el síndrome antisintetasa y las consideraciones anestésicas, se decidió seguir las directrices anestésicas descritas para las miopatías.

Se deben tener en cuenta las consideraciones preoperatorias, intraoperatorias y postoperatorias de estos pacientes, con el fin de evitar posibles complicaciones ${ }^{5}$.

Es importante hacer una buena evaluación preoperatoria ${ }^{5}$, añadiendo a las pruebas rutinarias una especial atención a la afectación muscular, pudiendo realizar un electromiograma, pruebas de función respiratoria y pruebas de función cardíaca, en función del estado general del paciente ${ }^{6}$.

Durante el intraoperatorio, la técnica anestésica de elección sería la anestesia regional, siempre que esta sea posible ${ }^{6}$. De no ser posible, como en nuestro caso, se deberán tener en cuenta los aspectos que minimicen el riesgo de complicaciones? ${ }^{7}$.

Los principales grupos farmacológicos a los que debemos prestar especial atención son los opioides, por su potencial efecto depresor respiratorio, y a los relajantes musculares, ya que pueden actuar de manera incierta en pacientes con miopatías. En este caso, se optó por la colocación de un catéter epidural para minimizar la dosis de opioides y asociar a su vez lidocaína tópica a la vía aérea para minimizar el efecto de la intubación ${ }^{7}$. El catéter epidural tiene interés tanto a nivel intraoperatorio como a nivel postoperatorio para poder tener un manejo analgésico con ausencia o bajas dosis de opioides, y de esta manera disminuir el riesgo de depresión respiratoria.

En cuanto al mantenimiento de la anestesia, en nuestro caso se optó por desfluorano para potenciar el efecto relajante del rocuronio, aunque no hay evidencia de ser superior al uso de propofol. Remifentanilo en combinación con el catéter epidural para la analgesia intraoperatoria, por la corta duración de acción de este. Es importante recordar que, en caso de ser necesaria la utilización de relajantes neuromusculares, es conveniente evitar los agentes despolarizantes como la succinilcolina y utilizar relajantes musculares no despolarizantes a dosis bajas y siempre bajo monitorización neuromuscular debido a la variabilidad en la respuesta. Los agentes despolarizantes tienen mayor riesgo de producir una respuesta miotónica que comprometa la ventilación. En este caso se decidió el empleo de bajas dosis de rocuronio, y el empleo de sugammadex para la reversión del bloque neuromuscular?
En cuanto al periodo postoperatorio es conveniente trasladar a estos pacientes a una unidad de reanimación para continuar con los cuidados postoperatorios y vigilar la presencia de posibles complicaciones, poniendo especial atención a la analgesia y la mecánica respiratoria. En el caso de nuestro paciente su estancia en reanimación fue de 24 horas sin presentar complicaciones durante su estancia en esta, ni durante su estancia en la planta, pudiendo ser dada de alta a los 5 días.

Este caso ilustra el manejo anestésico y perioperatorio de un paciente con síndrome antisintetasa, una entidad poco frecuente que puede conllevar diversos problemas intraoperatorios por su potencial afectación muscular, pulmonar y cardíaca.

\section{Conflicto de intereses}

Los autores declaran no tener ningún conflicto de interés

\section{Financiamiento}

Los autores declaran no haber recibido becas 0 ayuda.

\section{Responsabilidades éticas}

Protección de personas y animales. Los autores declaran que para esta investigación no se han realizado experimentos en seres humanos ni en animales.

Confidencialidad de los datos. Los autores declaran que han seguido los protocolos de su centro de trabajo sobre la publicación de datos de pacientes.

Derecho a la privacidad y consentimiento informado. Los autores han obtenido el consentimiento informado de los pacientes y/o sujetos referidos en el artículo. Este documento obra en poder del autor de correspondencia.

\section{Bibliografía}

1. Macias Fernandez I, Perez Venegas JJ, García Pérez S. Síndrome Antisintetasa. Semin Fund Esp Reumatol. 2007;8:28-3.

2. Cojocaru M, Cojocaru I, Chicos B. New insights into Antisynthetase Syndrome. Maedica (Buchar). 2016;11(2):130-5.

3. Lioger B, Lavigne C, Machet L. Dermatomiositis. EMC. Dermatología; 2011;1-12 [Article 98-500-A-10].

4. Toujani S, Amani Ben Mansour, Mjid M, Hedhli A, Cherif J, Ouahchy Y, Beji M. Acute respiratory failure as the first manifestation of antisynthetase syndrome. Tanaffos. 2017;16(1):76-9.

5. Cordero Escobar I. Consideraciones anestésicas en algunas enfermedades neuromusculares. Rev Mex Anest. 2009;32(2):114-9.

6. Cirpe. Web de cirugía y anestesiología infantil (Internet). Cassini EA. Miopatías y anestesia. Disponible en: http://163.178.103.176/CasosBerne/1aFCelular/Caso2-2/HTMLC/CasosB2/1/MIOPATIAS\%20Y\%20 ANESTESIA.htm

7. Kendigelen P, Cigdem Tutuncu A, Ashyralyyeva G, Hamamcioglu EA, Kaya G. Sugammadex usage in a patient with dermatomyositis. J Clin Anest. 2015;27(5):438-9. 